\title{
Renormalon Model of Twist-4 Corrections to the Pion Distribution Amplitude
}

\author{
JePpe R. ANDERSEN* \\ The Niels Bohr Institute \\ Blegdamsvej 17 \\ DK-2100 Copenhagen \\ Denmark
}

19th January 2000

\begin{abstract}
In this paper we describe a renormalon-inspired model of twist-4 power corrections to the light-cone pion distribution amplitude, and compare it to the results obtained using the conformal wave expansion. We find that the overall functional form predicted in this renormalon model is similar to the one predicted in the standard approach to higher twist contributions involving an expansion in conformal operators. However, small discrepancies at the end-points allow for a discussion on the convergence properties of the conformal expansion.
\end{abstract}

NBI-HE-99-33

*andersen@nbi.dk 


\section{Introduction}

Hadron distribution amplitudes play a key role in the study of hard exclusive processes in QCD. At large $Q^{2}$, amplitudes for such processes can be written as a convolution of a process dependent hard scattering amplitude which is calculable in perturbation theory, and a process independent non-perturbative distribution amplitude, one for each hadron participating in the process [1]. The leading twist-2 distribution amplitude for the pion at asymptotically large $Q^{2}$ is given by

$$
\phi(u)=6 u(1-u),
$$

to which there are corrections at finite $Q^{2}$ in the form of Gegenbauer polynomials. It is, however, generally believed that there will be large power suppressed corrections to the usual factorisation at accessible energies. For some observables (eg. the pion transition form factor for the process $\pi \rightarrow \gamma^{*} \gamma^{*}$ and light-cone sum rules for the decay $B \rightarrow \pi e \nu$ ), these power suppressed contributions are calculable in terms of process independent higher twist distribution amplitudes coming from higher Fock states and transverse momentum of the quarks. However, for eg. the pion form factor one has to also take into account so-called soft contributions (see eg. [2, 3]) that generally result in power corrections to the process dependent hard scattering amplitudes. This paper will not consider the problem of factorisation at the power suppressed levels or the soft contributions. Instead, we will focus on the higher twist distribution amplitudes from transverse momentum and Fock states with different spin components.

A gauge-invariant way to study the expansion of the pion distribution amplitude in terms of increasing twist is to consider the vacuum-to-pion matrix element. We parametrise it as [4]

$$
\begin{aligned}
& \left\langle 0\left|\bar{d}(0) \gamma_{\mu} \gamma_{5}[0, x] u(x)\right| \pi^{+}(p)\right\rangle \\
= & i f_{\pi} p_{\mu} \int_{0}^{1} \mathrm{~d} u e^{-i u p x}\left(\phi(u)+x^{2} g_{1}(u)+O\left(x^{4}\right)\right) \\
+ & f_{\pi}\left(x_{\mu}-\frac{x^{2} p_{\mu}}{p x}\right) \int_{0}^{1} \mathrm{~d} u e^{-i u p x} g_{2}(u)+\cdots,
\end{aligned}
$$

where $\phi(u)$ is the asymptotic leading twist contribution (11) and $[0, x]$ denotes the path-ordered gauge factor along the straight line between 0 and $x$

$$
[x, y]=P \exp \left[i g \int_{0}^{1} \mathrm{~d} t(x-y)_{\mu} A^{\mu}(t x+(1-t) y)\right] .
$$

$g_{1}(u)$ and $g_{2}(u)$ parametrise the twist-4 part and correspond to the contributions from the transverse momentum of the quarks and from states of the quarks with a different spin projection $\bar{\psi} \gamma_{-} \psi$, respectively.

The standard procedure for estimating higher twist contributions is to exploit a connection between $g_{1}(u), g_{2}(u)$ and the three-particle amplitudes. This connection is derived using the QCD equations of motion and allow one to calculate $g_{1}(u)$ and $g_{2}(u)$ through an expansion in conformal operators of the three-particle operator. It is generally believed that the anomalous dimensions of operators increase with increasing conformal spin, and so only a few terms in this expansion should be needed to get reliable results. The analysis in [4] includes the contribution to twist -4 distribution amplitudes from the operators of lowest and next-to lowest conformal spin. In this framework there has been recent progress in the study of pre-asymptotic behaviour of 
the meson distribution amplitudes, extending the analysis to vector mesons and including masscorrections [5, [5]. The results are being extensively used, e.g. in light-cone sum rule calculations of B-meson decay form factors [7] etc.

In the following we will describe a different approach that does not rely on the hierarchy of anomalous dimensions and therefore allows us to get some insight into the convergence properties of the conformal expansion.

\section{Light cone pion distribution amplitudes}

The relation (2) of the vacuum-to-pion transition amplitude has to be understood as an operator product expansion taken at tree level. At higher orders there will be corrections to eg. the coefficient of $\phi(u)$, which is taken to unity in (2). A more complete form of (2) to twist-4 accuracy is, therefore, (gauge-factors are implicit)

$$
\begin{aligned}
& \left\langle 0\left|\bar{d}(0) \gamma_{\mu} \gamma_{5} u(x)\right| \pi^{+}(p)\right\rangle \\
= & i f_{\pi} p_{\mu} \int_{0}^{1} \mathrm{~d} u e^{-i u p x}\left[\sum_{n=0} r_{n}\left(u, \mu^{2}\right) \alpha_{s}^{n}\left(\mu^{2}\right) \phi\left(u, \mu^{2}\right)+x^{2} g_{1}\left(u, \mu^{2}\right)\right] \\
+ & f_{\pi}\left(x_{\mu}-\frac{x^{2} p_{\mu}}{p x}\right) \int_{0}^{1} \mathrm{~d} u e^{-i u p x} g_{2}\left(u, \mu^{2}\right),
\end{aligned}
$$

where the distribution amplitudes $\phi(u), g_{1}(u)$ and $g_{2}(u)$ are defined as matrix elements of nonlocal quark-antiquark operators at strictly light-like separation. The projection on the lightcone introduces divergences, and the distribution amplitudes thus have to be renormalised. We choose the $\overline{\mathrm{MS}}$ subtraction scheme and the normalisation scale $\mu^{2}=1 / x^{2}$ so that the coefficient functions are free from logarithms of the type $\log \left(x^{2} \mu^{2}\right)$.

It is generally believed that the perturbative series $\sum_{n} r_{n} \alpha_{s}^{n}$ will have factorially divergent coefficients $r_{n}$ [8]. This introduces ambiguities in the definition of the sum of the series, usually referred to as infrared renormalon ambiguities. These ambiguities are artifacts of the perturbative factorisation, and for a physically observable transition amplitude these divergences must cancel with the corresponding ultraviolet renormalon ambiguities in the definition of the higher twist distribution amplitudes. In a different language, the renormalon problem indicates a double counting of the regions of small momenta if perturbation theory is not calculated with an explicit IR cutoff, and the double counting is reflected by quadratic divergences of the twist-4 operators. We refer the reader to Ref. [8] for the thorough discussion. The higher-twist contributions are, therefore, necessary to render the perturbative treatment of physical observables well defined. It follows that the ambiguities of the leading-twist perturbative series can be taken as a "minimal model" of higher-twist distribution amplitudes. This we will call the renormalon model.

Instead of calculating the higher-order contributions to the leading-twist coefficient function directly, it is more instructive to consider the ultraviolet renormalon ambiguity in twist-4 operators, which is equivalent according to the above argument. The calculation of the ultraviolet renormalon ambiguity in the twist-4 operators is similar to a calculation by L. Magnea, V.M. Braun and M. Beneke reported in [8]. As in the standard approach, it is convenient to study first the three-particle quark-antiquark-gluon operators and use the equations of motion. Specifically, the following identities, derived from the equations of motion for QCD string 
operators [9], can be used:

$$
\begin{aligned}
\frac{\partial}{\partial x_{\mu}} \bar{d}(-x) \gamma_{\mu} \gamma_{5} u(x) & =i \int_{-1}^{1} \mathrm{~d} v v \bar{d}(-x) x_{\alpha} g G_{\alpha \beta}(v x) \gamma_{\beta} \gamma_{5} u(x), \\
\partial_{\mu}\left\{\bar{d}(-x) \gamma_{\mu} \gamma_{5} u(x)\right\} & =i \int_{-1}^{1} \mathrm{~d} v \bar{d}(-x) x_{\alpha} g G_{\alpha \beta}(v x) \gamma_{\beta} \gamma_{5} u(x) .
\end{aligned}
$$

Here $\partial_{\mu}$ is the derivative with respect to a translation of the nonlocal operator, while $\frac{\partial}{\partial x_{\mu}}$ is the derivative with respect to the interquark separation. $G_{\alpha \beta}$ is the gluon field strength tensor. The three-particle light-cone distribution amplitudes on the right hand side can be parametrised as [4]

$$
\begin{aligned}
& \left\langle 0\left|\bar{d}(-x) \gamma_{\mu} \gamma_{5} G_{\alpha \beta}(v x) u(x)\right| \pi^{+}(p)\right\rangle \\
= & p_{u}\left(p_{\alpha} x_{\beta}-p_{\beta} x_{\alpha}\right) \frac{1}{p x} f_{\pi} \int \mathscr{D} \alpha_{i} \Phi_{\|}\left(\alpha_{i}\right) e^{-i p x\left(\alpha_{1}-\alpha_{2}+v \alpha_{3}\right)} \\
+ & {\left[p_{\beta}\left(g_{\alpha \mu}-\frac{x_{\alpha} p_{\mu}}{p x}\right)-p_{\alpha}\left(g_{\beta \mu}-\frac{x_{\beta} p_{\mu}}{p x}\right)\right] f_{\pi} \int \mathscr{D} \alpha_{i} \Phi_{\perp}\left(\alpha_{i}\right) e^{-i p x\left(\alpha_{1}-\alpha_{2}+v \alpha_{3}\right)}, }
\end{aligned}
$$

The operator on the left hand side contains contributions of both twist -3 and twist -4 , but the matrix element of the twist -3 part vanishes. The pion decay constant $f_{\pi}$ is defined through

$$
\left\langle 0\left|\bar{u}(0) \gamma_{\mu} \gamma_{5} d(0)\right| \pi^{+}(P)\right\rangle=i f_{\pi} P_{\mu}
$$

and the integration measure on the right hand side is defined as

$$
\int \mathscr{D} \alpha_{i}=\int_{0}^{1} \mathrm{~d} \alpha_{1} \mathrm{~d} \alpha_{2} \mathrm{~d} \alpha_{3} \delta\left(1-\alpha_{1}-\alpha_{2}-\alpha_{3}\right) .
$$

By sandwiching (5) and (6) between the vacuum and pion state it is now possible to derive the following relations between the parameterisations of the twist-4 distribution amplitudes and the three particle operator [4]

$$
\begin{aligned}
g_{2}(u) & =\int_{0}^{u} \mathrm{~d} \alpha_{1} \int_{0}^{\bar{u}} \mathrm{~d} \alpha_{2} \frac{1}{\alpha_{3}}\left[2 \Phi_{\perp}-\Phi_{\|}\right]\left(\alpha_{1}, \alpha_{2}, \alpha_{3}\right), \\
g_{1}(u)+\int_{0}^{u} \mathrm{~d} v g_{2}(v) & =\frac{1}{2} \int_{0}^{u} \mathrm{~d} \alpha_{1} \int_{0}^{\bar{u}} \mathrm{~d} \alpha_{2} \frac{1}{\alpha_{3}^{2}}\left(\bar{u} \alpha_{1}-u \alpha_{2}\right)\left[2 \Phi_{\perp}-\Phi_{\|}\right]\left(\alpha_{1}, \alpha_{2}, \alpha_{3}\right) .
\end{aligned}
$$

Here $\alpha_{3}=1-\alpha_{1}-\alpha_{2}$ and $\bar{u}=1-u$. In the following analysis we will calculate an explicit expression for the special combination $\left[2 \Phi_{\perp}-\Phi_{\|}\right]\left(\alpha_{1}, \alpha_{2}, \alpha_{3}\right)$ in the renormalon model and restore $g_{1}(u)$ and $g_{2}(u)$ from these expressions.

\section{The Renormalon Calculation}

It is generally believed that although the perturbative coefficients for QCD perturbative series show signs of factorial divergence, the series are asymptotic series for which we can assign a sum using the Borel transformation [8]. The Borel transform of a perturbative series

$$
R(a)=r_{0} a+r_{1} a^{2}+r_{2} a^{3}+\cdots=\sum_{n=0}^{\infty} r_{n} a^{n+1}
$$


is defined as

$$
B[R](t)=\sum_{n=0}^{\infty} t^{n} \frac{r^{n}}{n !}
$$

If $B[R](t)$ is well-defined on the positive real axis and is integrable we may define the Borel sum of the series as

$$
\tilde{R}=\int_{0}^{\infty} \mathrm{d} t e^{-t / a} B[R](t),
$$

with a series expansion identical to that of $R$. For convergent series, the Borel sum is therefore identical to the sum of the original series, and we can regard the Borel sum as an extension of the definition of the sum of a series to divergent, asymptotic series.

However, for a QCD observable $R$ the Borel transform $B[R]$ has singularities on the real axis, leading to ambiguities in the definition of the integral (13). These so-called renormalons are a direct result of the divergence of the original series, and as already mentioned the residues of the renormalon poles are taken as an estimate of the non-perturbative contribution to the observable [8]. For QCD observables, one finds that there are infrared renormalons stemming from the low-momentum region of higher order loop contributions, situated at $w=t / \beta_{0}=$ $1,2, \ldots$ Here $\beta_{0}$ is the first $\beta$-function coefficient $\frac{\mathrm{d} \alpha_{s}}{\mathrm{~d} \ln \mu^{2}}=-\beta_{0} \alpha_{s}^{2}+\cdots$

As seen from (13), the contribution from a pole situated at $w_{i}$ in the Borel plane to the observable will be suppressed by $\exp \left(-w_{i} \beta_{0} / \alpha_{s}\right)$. As the QCD coupling (at one-loop approximation) fulfils $1 / \alpha_{s}=\beta_{0} \ln \left(Q^{2} / \Lambda^{2}\right)$, we will need the IR renormalon at $w=1$ to obtain the $1 / Q^{2}$ twist -4 corrections.

It is certainly impossible to calculate the residue of the Borel transform at a given pole exactly, since that would require full knowledge of all the perturbative coefficients in the series. Instead we will apply the so-called Naive non-Abelianization (NNA) [10, 11, 12, 13] in which the coefficients are approximated by their leading $\beta_{0}$ part. The NNA starts from the approximation of the perturbative coefficients with the part coming from diagrams with one string of fermion bubbles inserted to a gluon line. In this way the leading $N_{f}$ piece $\left(N_{f}\right.$ being the number of quark flavours) is found. The full one-loop $\beta$ function behaviour is then restored by substituting for $N_{f}$ the scaled $\beta$-function coefficient $N_{f}-\frac{33}{2}$. This results in a gauge-invariant approximation of the diagrams to all orders in the coupling [8]. The positions of the poles in the Borel plane do not depend on the higher $\beta$-function coefficients and so are unchanged in the NNA compared to the full theory. On the other hand, the residues change and one actually finds that also the nature of the divergences may change. In this way, the pole-divergence of the Borel transform at $w=1$ in the NNA is actually the end-point of a branch cut in the full theory.

The NNA is useful for doing calculations, since in this approximation the Borel transformation applies to the modified gluon propagator itself rather than the diagram as a whole. The Borel-transformed effective gluon propagator in the Lorentz gauge is given by [8]

$$
B\left[\alpha_{s}\left(\mu^{2}\right) D_{\mu \nu}\right](w)=\frac{-i}{k^{2}}\left(g_{\mu \nu}-\frac{k_{\mu} k_{\nu}}{k^{2}}\right)\left(-\frac{\mu^{2}}{k^{2}} e^{-C}\right)^{w},
$$

where the $k_{\mu} k_{\nu}$-terms can be neglected for gauge-invariant quantities. $\mu$ is the renormalisation scale and $C$ is a renormalisation scheme-dependent constant. In the $\overline{\mathrm{MS}}$-scheme $C=-5 / 3$. It is noted that the only essential difference between (14) and the original gluon propagator is the 


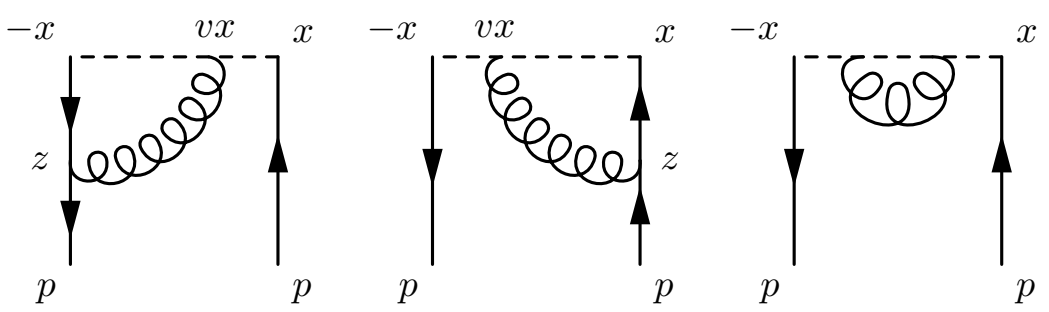

Figure 1: The twist-4 contributions to the renormalisation of the three-particle operator (7). The third diagram is scale-less and vanishing $[\mathbb{8}]$.

power of $k^{2}$ in the denominator, and therefore a calculation of the Borel transform of a diagram in the NNA to all orders in the coupling is no more difficult than the calculation of the same diagram with a simple gluon propagator.

We start by noting that to project out the the sought combination $\left[2 \Phi_{\perp}-\Phi_{\|}\right]$, we have to contract the three-particle operator in (7) with $\left(-x^{\alpha} g^{\mu \beta}\right)$. We want to calculate the leading ultraviolet renormalon singularity in the matrix element of this operator at $w=1$. Since this singularity comes from contributions of large momenta, the particular external states do not matter and it is sufficient to evaluate the contribution of the diagrams in Fig. 1 between quark states.

In the Borel representation the matrix element of the projected operator in (7) between quark states of momentum $p$ is given by

$$
\begin{aligned}
& \left\langle p\left|\bar{d}(-x) \gamma_{\mu} \gamma_{5} G_{\alpha \beta}(v x) u(x) x^{\alpha} g^{\mu \beta}\right| p\right\rangle \\
= & i 4 \pi C_{F}\left(\mu^{2} e^{-C}\right)^{w} \bar{d}(p) e^{-2 i p x}\left(g^{\lambda} x^{\nu}-x_{\mu} g^{\lambda \nu}\right) \\
\cdot & \left\{\gamma^{\mu} \gamma^{\sigma} \gamma_{\lambda} \gamma_{5} \int \frac{\mathrm{d}^{4} k}{(2 \pi)^{4} i} e^{-i \tilde{v} k x} \frac{k_{\nu}(p+k)_{\sigma}}{\left(-k^{2}\right)^{w+1}\left[-(p+k)^{2}\right]}\right. \\
+ & \left.\gamma_{\lambda} \gamma_{5} \gamma^{\sigma} \gamma^{\mu} \int \frac{\mathrm{d}^{4} k}{(2 \pi)^{4} i} e^{i \bar{v} k x} \frac{k_{\nu}(p-k)_{\sigma}}{\left(-k^{2}\right)^{w+1}\left[-(p-k)^{2}\right]}\right\} u(p),
\end{aligned}
$$

where $\bar{v}=1-v$. For $x$ strictly light-like, we find for the Borel-transformed operators in a neighbourhood of $w=1$

$$
\begin{array}{ll} 
& \bar{d}(-x) \gamma_{\mu} \gamma_{5} G_{\alpha \beta}(v x) u(x) x^{\alpha} g^{\mu \beta} \\
\begin{array}{c}
= \\
\text { quad. diverg. }
\end{array} & 2 i(4 \pi) C_{F} \mu^{2} e^{-C} \frac{1}{32 \pi^{2}} \frac{1}{w-1} \\
& \cdot \int_{0}^{1} \mathrm{~d} \alpha \alpha\left\{\bar{d}[-x] \gamma_{5} \not u[x(1-\alpha \bar{v})]-\bar{d}[-x(1-\alpha \tilde{v})] \gamma_{5} \nsim u[x]\right\} .
\end{array}
$$

As already mentioned, the quadratic ultraviolet divergence of the residue of the pole is independent of the external states, which allows us to write the relation as an operator relation. Note by comparing (16) and (2) that we have written the twist-4 operator as an integral over leading twist contributions. By taking the residue at $w=1$ of the pion to vacuum transition amplitude of the twist- 4 operator we find

$$
\left[2 \Phi_{\perp}-\Phi_{\|}\right]\left(\alpha_{i}\right)=-C_{F} \mu^{2} e^{-C} \frac{1}{4 \pi}\left\{\frac{\alpha_{3}}{\left(1-\alpha_{2}\right)^{2}} \phi\left(1-\alpha_{2}\right)-\frac{\alpha_{3}}{\left(1-\alpha_{1}\right)^{2}} \phi\left(\alpha_{1}\right)\right\},
$$


Choosing the asymptotic expression for the pion distribution amplitude $\phi(u)$ in (17), and inserting this result in (10) we obtain the following explicit expressions for the distribution amplitudes $g_{1}(u), g_{2}(u)$ :

$$
\begin{aligned}
g_{2}(u)= & N(u \log u-\bar{u} \log \bar{u}) \\
g_{1}(u)= & N \frac{1}{4}(-2 \bar{u} u+2 \log u \log \bar{u}+2 \bar{u} \log \bar{u}+2 u \log u \\
& \left.-\bar{u} \log ^{2} \bar{u}-u \log ^{2} u-2 u \operatorname{Li}_{2}\left(\frac{-\bar{u}}{u}\right)-2 \bar{u} \operatorname{Li}_{2}\left(\frac{-u}{\bar{u}}\right)\right) . \\
N= & \frac{3}{2 \pi} C_{F} \mu^{2} e^{-C}
\end{aligned}
$$

with $\mathrm{Li}_{2}(z)$ the di-logarithm.

\section{Discussion}

Unfortunately, the overall normalisation of the distribution amplitudes $g_{1}(u), g_{2}(u)$ is not accessible from the renormalon model and has to be found from eg. the QCD sum rule method. This is also the case for renormalon models of DIS structure functions and the conformal approach to pion distribution amplitudes.

However, the normalisation factor $\mathrm{N}$ found using the renormalon residue can be taken to indicate the order of magnitude of the twist- 4 correction. Using $\mu=\Lambda=0.250 \mathrm{GeV}$ we get $N \approx 0.091 \mathrm{GeV}^{2}$. Thereby we find $\int_{0}^{1} g_{1}(u) \mathrm{d} u=0.0088 \mathrm{GeV}^{2}$ which has to be compared with the result obtained by normalising the conformal results using light-cone QCD sum rules. Using the functional form and normalisation for the conformal approach of Ref. [4] we find $\int_{0}^{1} g_{1}(u) \mathrm{d} u=$ $0.028 \mathrm{GeV}^{2}$. For the first moment of the distribution amplitude $g_{2}$ we find $\int_{0}^{1} \mathrm{~d} u(2 u-1) g_{2}(u)=$ $0.0051 \mathrm{GeV}^{2}$ and $\int_{0}^{1} \mathrm{~d} u(2 u-1) g_{2}(u)=0.011 \mathrm{GeV}^{2}$ for the renormalon and conformal approach respectively.

It is more interesting that the shapes of the predicted wave amplitudes are close to the ones obtained in the conformal approach of [4], see Fig. 2. The functions have been normalised so that $\int_{0}^{1} \mathrm{~d} u g_{1}(u)=1$ and $\int_{0}^{1} \mathrm{~d} u(2 u-1) g_{2}(u)=1$. The similarity is encouraging, since it indicates that the approximations are well understood. The somewhat broader shape of the distribution amplitude $g_{1}(u)$ in the renormalon model compared to the conformal expansion is explained by the contributions of higher conformal spin operators. The anomalous dimensions of operators are ignored in the NNA approximation, so that it effectively corresponds to the summation of the conformal series to all orders, including, however, all kinematic suppression factors. The renormalon model, therefore, pretends to make a simple estimate of higher orders in the conformal expansion, and the main result of our study is that such contributions seem to be comfortably small, apart from in the end-point regions. The asymptotic behaviour of the renormalon model prediction for the distribution amplitude $g_{2}(u)$ at $x \rightarrow 0,1$ coincides with that of the lowest conformal spin contribution to $g_{2}(u)$. At the same time, the behaviour of $g_{1}(u)$ is different. The renormalon result behaves as $u$ for $u \rightarrow 0$ compared to the $u^{2}$ behaviour of each conformal operator, indicating that the series in conformal operators is not converging uniformly. This feature will have an effect on observables sensitive to these regions like $B$-meson decay form factors []]. 

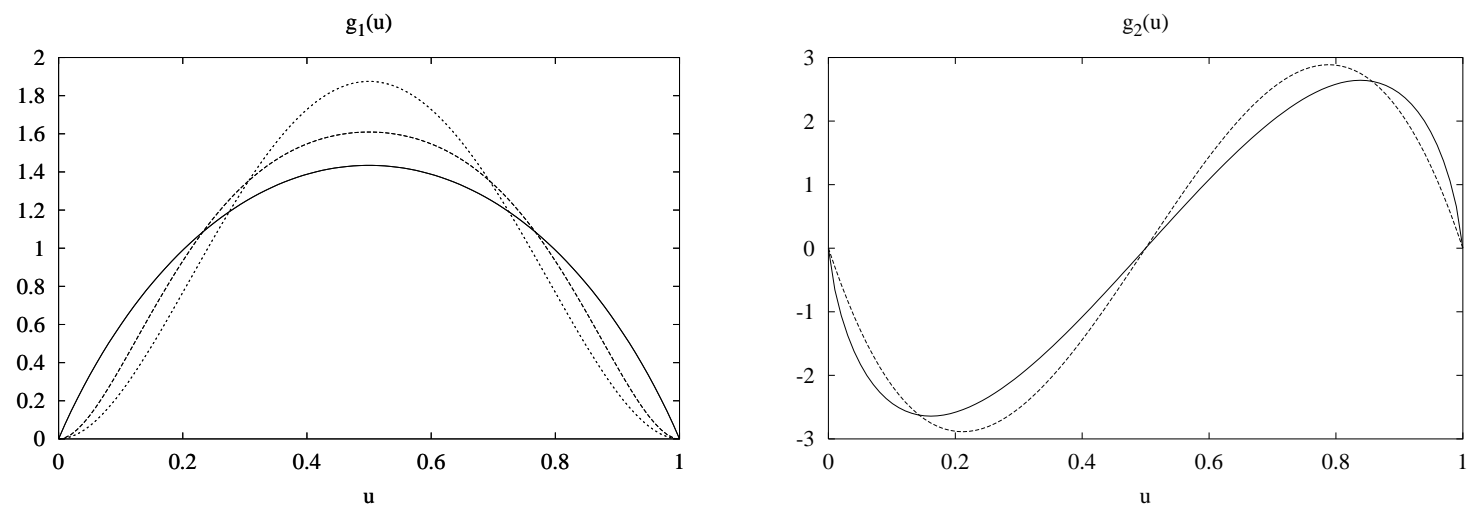

Figure 2: $g_{1}(u)$ (left) and $g_{2}(u)$ (right) calculated in both the renormalon model (line) and the conformal wave expansion of [4] (dots for the lowest conformal spin result and dashes for the result including next-to-lowest conformal spin operators). The distribution amplitudes have been normalised so that $\int_{0}^{1} \mathrm{~d} u g_{1}(u)=1$ and $\int_{0}^{1} \mathrm{~d} u(2 u-1) g_{2}(u)=1$.

\section{Acknowledgements}

The author is grateful to V.M. Braun for introducing him to the subject and for countless valuable discussions. Also, the Lørup Foundation is acknowledged for financial support for a stay in Regensburg during the final stages of this work and for participation in the workshop "Light-Cone Wave Functions in QCD" in Regensburg.

\section{References}

[1] G. Peter Lepage and Stanley J. Brodsky. Exclusive processes in perturbative quantum chromodynamics. Phys. Rev., D22:2157, 1980.

[2] S. S. Agaev. Electromagnetic pion form-factor in the context of the principal value method. Phys. Lett., B360:117-122, 1995.

[3] Vladimir Braun and Igor Halperin. Soft contribution to the pion form-factor from light cone QCD sum rules. Phys. Lett., B328:457-465, 1994.

[4] V. M. Braun and I. E. Filyanov. Conformal invariance and pion wave functions of nonleading twist. Z. Phys., C48:239-248, 1990.

[5] Patricia Ball, V. M. Braun, Y. Koike, and K. Tanaka. Higher twist distribution amplitudes of vector mesons in QCD: Formalism and twist three distributions. Nucl. Phys., B529:323, 1998.

[6] Patricia Ball and V. M. Braun. Higher twist distribution amplitudes of vector mesons in QCD: Twist - 4 distributions and meson mass corrections. Nucl. Phys., B543:201, 1999.

[7] Patricia Ball and V. M. Braun. Exclusive semileptonic and rare B meson decays in QCD. Phys. Rev., D58:094016, 1998. 
[8] M. Beneke. Renormalons. Phys. Rept., 317:1, 1999.

[9] I. I. Balitsky and V. M. Braun. Evolution equations for QCD string operators. Nucl. Phys., B311:541-584, 1989.

[10] D. J. Broadhurst and A. G. Grozin. Matching QCD and HQET heavy - light currents at two loops and beyond. Phys. Rev., D52:4082-4098, 1995.

[11] M. Beneke and V. M. Braun. Naive nonAbelianization and resummation of fermion bubble chains. Phys. Lett., B348:513-520, 1995.

[12] Patricia Ball, M. Beneke, and V. M. Braun. Resummation of $\left(\beta_{0} \alpha_{s}\right)^{n}$ corrections in QCD: Techniques and applications to the $\tau$ hadronic width and the heavy quark pole mass. Nucl. Phys., B452:563-625, 1995.

[13] C. N. Lovett-Turner and C. J. Maxwell. All orders renormalon resummations for some QCD observables. Nucl. Phys., B452:188-212, 1995. 